\title{
Evolución clínica de la estenosis aórtica: cuando el flujo marca la diferencia
}

\section{Outcome in Aortic Stenosis: When the Flow Makes the Difference}

\author{
Patrizio Lancellotti* y Raluca Dulgheru \\ University of Liege Hospital, GIGA Cardiovascular Sciences, Heart Valve Clinic, Department of Cardiology, CHU Sart Tilman, Lieja, Bélgica
}

Historia del artículo:

On-line el 8 de febrero de 2013

Inicialmente, la estenosis aórtica (EA) se describió como una enfermedad aislada que afectaba a las valvas de la válvula aórtica. Pero en los últimos años este concepto ha evolucionado con cambios importantes, y actualmente se considera a la EA una enfermedad "sistémica» compleja ${ }^{1}$. Gran parte de nuestro conocimiento actual sobre la EA procede de estudios que demuestran que los pacientes con EA degenerativa no tienen una función arterial normal. Entre estos pacientes hay mayor prevalencia de aterosclerosis, hipertensión sistólica aislada y enfermedad coronaria; su ventrículo izquierdo (VI) debe superar no solamente la obstrucción valvular, sino también la carga hemodinámica arterial elevada resultante ${ }^{2-5}$. En última instancia, la gravedad de la EA como enfermedad sistémica se refleja en cómo el VI logra superar tanto el problema valvular como la carga hemodinámica arterial. En respuesta al aumento de la carga hemodinámica total del VI, este tiene que generar una presión superior por mililitro de sangre para expulsarla durante la sístole. Sin embargo, cuando se agotan los mecanismos de adaptación, el VI es incapaz de mantener un flujo suficiente a través de la válvula aórtica. En consecuencia, deja de ser posible satisfacer las necesidades metabólicas tisulares y pueden aparecer los síntomas.

Esta teoría tiene varias consecuencias. En primer lugar, puede explicar por qué un paciente con una EA sólo moderada pero con hipertensión concomitante y baja distensibilidad arterial sistémica puede presentar síntomas similares a los de una EA grave (disnea de esfuerzo, síncope o angina) o mostrar una reducción de la fracción de eyección del VI (FEVI) $(<50 \%)$. En segundo lugar, llama la atención respecto al hecho de que el flujo existente a través de la válvula aórtica es importante para estratificar la gravedad de la EA. De hecho, refleja de manera aproximada la compleja interrelación existente entre la función de bombeo del VI, el grado de disfunción de la válvula aórtica y la función arterial. Así pues, en los pacientes con EA, se considera que es un aspecto crucial examinar el flujo a través de la válvula aórtica.

La decisión sobre el modo de tratamiento que se debe utilizar se basa en el estado sintomático y la gravedad de la enfermedad de los pacientes con EA. Las guías actuales recomiendan la sustitución de la válvula aórtica, con una indicación de clase I, sólo para los pacientes sintomáticos se considera tienen una EA grave ${ }^{6,7}$. En la

VÉASE CONTENIDO RELACIONADO:

http://dx.doi.org/10.1016/j.recesp.2012.09.006, Rev Esp Cardiol. 2013;66:261-8.

* Autor para correspondencia: Department of Cardiovascular Imaging, University Hospital Sart Tilman, Bâtiment B 35-4000 Lieja, Bélgica.

Correo electrónico: plancellotti@chu.ulg.ac.be (P. Lancellotti).
EA moderada, la indicación de la sustitución de la válvula aórtica es de clase Ila y está sujeta a que se practique al paciente una cirugía de revascularización aortocoronaria, cirugía de la aorta ascendente $\mathrm{u}$ otra válvula. Por consiguiente, es imprescindible establecer exactamente la gravedad de la EA para la toma de decisiones clínicas. La EA grave suele definirse de la siguiente forma: área valvular aórtica (AVA) $<1 \mathrm{~cm}^{2}$, gradiente de presión transaórtico medio $>40 \mathrm{mmHg}$ y velocidad máxima del chorro (jet) aórtico $>4 \mathrm{~m} / \mathrm{s}$. Hasta la fecha, ninguno de los parámetros definidos para determinar el grado de la EA se puede aplicar como criterio único para establecer su gravedad; cada uno tiene sus propias limitaciones. De hecho, con frecuencia se observan discrepancias entre el gradiente de presión transaórtico medio y el AVA en un mismo paciente. Estas discrepancias son fáciles de comprender en pacientes con bajo gasto cardiaco junto con reducción de la FEVI, pero pueden darse también en pacientes con función del VI aparentemente conservada ${ }^{1,8}$. En la práctica clínica diaria, estas discrepancias pueden llevar a subestimar la estenosis y la gravedad de los síntomas y, por lo tanto, a un retraso inadecuado de la sustitución de la válvula aórtica en pacientes con EA.

Los gradientes transvalvulares dependen en gran medida de la variación del flujo, puesto que son una función cuadrática del flujo que pasa por la válvula ${ }^{4,9-11}$. Una disminución tan sólo moderada del flujo puede conducir a una reducción significativa del valor medio o máximo del gradiente de presión. Un gradiente bajo, sobre todo en presencia de un flujo reducido por la válvula, no descarta por completo la presencia de una EA grave. Por el contrario, si se han descartado cuidadosamente los posibles errores de medición, un gradiente bajo puede ser útil para identificar a grupos de pacientes con EA grave, que tienen mayor riesgo de episodios cardiovasculares futuros ${ }^{11-14}$. Así pues, el análisis del flujo a través de la válvula es un paso crucial en la evaluación de la gravedad de la EA. El AVA se considera un parámetro menos dependiente del flujo en la evaluación de la gravedad de la EA, aunque el AVA sola no puede ser el único parámetro aplicado para estimar la gravedad de la EA. El AVA depende en gran manera de la medición exacta del área del infundíbulo de salida del VI, que constituye la principal fuente de error. Además, el valor de corte del AVA que indica una EA grave continúa siendo motivo de controversia; algunos autores sugieren un valor de corte de $0,8 \mathrm{~cm}^{2}$ como más adecuado, puesto que permitiría reconciliar las discordancias existentes con el gradiente de presión transaórtico medio ${ }^{8}$. Sin embargo, varios estudios en el pasado clasificaron la EA como grave con un valor de corte de AVA $<1 \mathrm{~cm}^{2}$, con independencia del gradiente de presión 
transaórtico medio, han puesto de manifiesto que este valor concreto tiene capacidad predictiva para detectar un exceso de mortalidad y morbilidad ${ }^{4,12}$. Así pues, un AVA $<1 \mathrm{~cm}^{2}$ es igual de concluyente para establecer el nivel de gravedad de la EA de los pacientes.

Recientemente, varios autores han resaltado que con la misma caracterización de la EA grave (AVA $<1 \mathrm{~cm}^{2}$ ) se puede identificar varias situaciones que difieren en su flujo transvalvular y su gradiente de presión ${ }^{4,9-11}$. El primer grupo que subrayó la importancia de integrar las relaciones válvula-gradiente con el patrón de flujo fue el de Pibarot et $\mathrm{al}^{4}$, mientras que Miners et $\mathrm{al}^{8}$ fueron los primeros en mostrar claramente las inconsistencias que comporta establecer el nivel de gravedad de la EA y que propusieron, junto con Lancellotti et $\mathrm{al}^{14}$, la nueva clasificación de la EA. En los pacientes con un AVA $<1 \mathrm{~cm}^{2}$, se puede identificar cuatro categorías de flujo-gradiente de la EA: flujo normal/ gradiente bajo (FN/GB), flujo normal/gradiente alto (FN/GA), flujo bajo/gradiente bajo (FB/GB) y flujo bajo/gradiente alto (FB/GA). El FB se define como un volumen de eyección del VI indexado $<5 \mathrm{ml} /$ $\mathrm{m}^{2}$; el $\mathrm{GB}$ se define como un gradiente de presión transaórtico medio $<40 \mathrm{mmHg}^{7}$.

El patrón FN/GB se observa en un 31-38\% de los pacientes y parece corresponder al grupo de pacientes con un nivel menos grave de EA (una inconsistencia intrínseca que está en las guías) o a los pacientes que han estado expuestos a la enfermedad menos tiempo. Esta entidad se caracteriza por una función miocárdica longitudinal del VI conservada, con menores concentraciones de péptido natriurético cerebral (BNP) y una puntuación de riesgo de Monin más baja ${ }^{14,15}$. El pronóstico de estos pacientes parece estar relativamente conservado en comparación con el de pacientes de otras categorías.

El patrón FN/GA corresponde al de mayor prevalencia (39-72\%) en pacientes con EA y concuerda plenamente con los criterios propuestos en las guías ${ }^{4,14-16}$. En comparación con el grupo FN/GB, aunque la función longitudinal del VI está conservada en estos pacientes, las cifras de BNP son más altas y las tasas de supervivencia sin eventos cardiacos, más bajas. Además, los pacientes con FN/GA parecen tener una EA más grave, lo que indica una exposición más prolongada a esta enfermedad progresiva. Cuando son sintomáticos, se suele remitirlos a la sustitución de la válvula aórtica; sin embargo, cuando son asintomáticos, el tratamiento de estos pacientes subraya la necesidad de una estratificación optimizada del riesgo.

El patrón $\mathrm{FB} / \mathrm{GA}$ se da en un $8 \%$ de los pacientes con EA grave ${ }^{4,14-16}$. Se caracteriza por un volumen de eyección del VI indexado $<35 \mathrm{ml} / \mathrm{m}^{2}$ a pesar de conservarse la FEVI, las cifras de BNP elevadas, una puntuación de riesgo de Monin alta y una reducción significativa de la función longitudinal del $\mathrm{VI}^{14}$. Es necesario señalar que la FEVI es una estimación bruta de la función sistólica del VI. La FEVI se ve influida por la función miocárdica intrínseca y por la geometría de la cavidad del VI. Así pues, para un grado similar de acortamiento miocárdico intrínseco, la FEVI tenderá a aumentar en relación con el grado de remodelado concéntrico del VI. Por tanto, la FEVI puede subestimar notablemente el grado de deterioro del miocardio en presencia de remodelado concéntrico del VI, como suele ocurrir en los pacientes con EA. Así pues, lo que es normal en cuanto a función sistólica para un VI de geometría normal, puede ser anormal para un VI con un remodelado concéntrico. Además, la reducción del gasto del VI (en relación con una disfunción miocárdica intrínseca y un importante remodelado del VI) puede conducir, a su vez, a unos gradientes transvalvulares inferiores a los esperados. La evolución clínica de la enfermedad en esos pacientes es casi idéntica a la de los pacientes con FN/GA. Cuando son sintomáticos, estos pacientes tienden a tener mejor supervivencia si se los trata quirúrgicamente.
La prevalencia del patrón $\mathrm{FB} / \mathrm{GB}$ parece ser inferior a la inicialmente descrita. Este patrón supone un 7\% del total de pacientes asintomáticos y un 15-35\% de los pacientes sintomáti$\cos ^{4,9-11}$. El patrón, que se designa como EA FB paradójica, es una entidad clínica difícil de abordar, cuya importancia se ha resaltado recientemente. Se asocia a un remodelado concéntrico del VI más pronunciado, una cavidad del VI más pequeña, aumento de la poscarga del VI total, disfunción miocárdica intrínseca, fibrosis miocárdica y pronóstico adverso ${ }^{9,10}$. En los pacientes asintomáticos con EA FB/GB, nuestro estudio puso de manifiesto que la probabilidad de continuar con vida sin tratamiento con sustitución de la válvula aórtica a los 3 años es 5 veces menor que la del grupo de pacientes FN/GB y 4 veces mayor que la del grupo de pacientes $\mathrm{FN} / \mathrm{GA}^{14}$. Esta entidad clínica con frecuencia se diagnostica erróneamente, lo que puede conducir a que se subestime la gravedad de la EA y a infrautilizar o retrasar inadecuadamente la cirugía. Es absolutamente crucial identificar esta entidad y asegurar que no se niega la cirugía a un paciente sintomático con AVA baja y GB. En la práctica clínica, cuando el AVA es $<1 \mathrm{~cm}^{2}$ y el gradiente de presión transaórtico medio es $<40 \mathrm{mmHg}$, es preciso descartar en primer lugar los errores de medición y el tamaño corporal pequeño (AVA indexada). A continuación se debe identificar las características típicas de una EA FB paradójica: índice de volumen de eyección $\leq 35 \mathrm{ml} / \mathrm{m}^{2}$ asociado a un VI reducido, un diámetro telediastólico $<47 \mathrm{~mm}$ y un índice de volumen $<55 \mathrm{ml} / \mathrm{m}^{2}$, aumento del grosor relativo de la pared $>0,45$ e impedancia valvuloarterial $>4,5$ $\mathrm{mmHg} / \mathrm{ml} / \mathrm{m}^{2}$.

En el artículo publicado en Revista Española de Cardiología, Melis et al, siguiendo la nueva clasificación propuesta para la EA grave según el patrón de flujo-gradiente, han realizado un análisis retrospectivo de la evolución clínica de los pacientes con EA grave $\left(\right.$ AVA $\left.<1 \mathrm{~cm}^{2}\right)$ y FEVI conservada $(\geq 50 \%)^{16}$. El objetivo de valoración principal fue la mortalidad total. El resultado principal de este estudio fue que los pacientes con EA FB grave, con independencia de la gradiente de presión transaórtico medio, tenían unas tasas de mortalidad superiores a las de los pacientes con EA FN (el 26,6 frente al 13,6\%; p =0,004). Además, el grupo FN/GB fue el de mejor pronóstico respecto a la mortalidad total. Estos resultados concuerdan con los de nuestro estudio ${ }^{14}$. Además, el porcentaje de pacientes de cada grupo en sus poblaciones de estudio fue similar a los resultados obtenidos en nuestra población; aproximadamente la mitad de la población tiene una EA FN/GA grave; alrededor de un $27 \%$ de la población, una EA $\mathrm{FN} / \mathrm{GB}$; un $15 \%$, una $\mathrm{EA} \mathrm{FB} / \mathrm{GA}$, y un $12 \%$, una $\mathrm{EA} \mathrm{FB} / \mathrm{GB}$ grave. La mayor prevalencia de $\mathrm{EA} \mathrm{FB} / \mathrm{GB}$ grave en el presente estudio en comparación con nuestros datos (el 12 frente al 7\%) probablemente se debiera a las diferencias en los criterios de inclusión de los pacientes (pacientes tanto sintomáticos como asintomáticos frente a pacientes realmente asintomáticos). En la misma línea que otras publicaciones, el artículo de Melis et al refuerza el hecho de que los pacientes con EA FB/GB grave tienen menor diámetro del VI, menor distensibilidad arterial sistémica, mayor resistencia vascular sistémica, mayor carga hemodinámica general (valvular y arterial) y peor evolución clínica ${ }^{4,14}$. El estudio tiene algunas limitaciones que es preciso reconocer: $a$ ) su carácter retrospectivo, y b) que la prevalencia de enfermedad coronaria fuera más frecuente en el grupo de pacientes con EA FB/GB grave, lo cual puede explicar en parte la mayor tasa de mortalidad de este grupo. Sin embargo, la mayor prevalencia de enfermedad coronaria en pacientes con EA FB/GB grave forma parte del carácter "sistémico» de esta enfermedad y no se la puede considerar un parámetro de diferenciación concluyente.

La clasificación de una EA grave asintomática con el empleo del nuevo sistema propuesto, teniendo en cuenta el AVA y los cuatro patrones de flujo-gradiente, podría reducir el grado de incertidumbre asociado a la interpretación de la gravedad de esta 
valvulopatía en la práctica clínica. Este enfoque es unificador y permite una mejor caracterización de la evolución clínica de estos pacientes.

\section{CONFLICTO DE INTERESES}

Ninguno.

\section{BIBLIOGRAFÍA}

1. Pibarot P, Dumesnil JG. Improving assessment of aortic stenosis. J Am Coll Cardiol. 2012;60:169-80.

2. Briand M, Dumesnil JG, Kadem L, Tongue AG, Rieu R, Garcia D, et al. Reduced systemic arterial compliance impacts significantly on left ventricular afterload and function in aortic stenosis: implications for diagnosis and treatment. J Am Coll Cardiol. 2005;46:291-8.

3. Lancellotti P, Donal E, Magne J, Moonen M, O'Connor K, Daubert JC, et al. Risk stratification in asymptomatic moderate to severe aortic stenosis: the importance of the valvular, arterial and ventricular interplay. Heart. 2010;96:1364-71.

4. Hachicha Z, Dumesnil JG, Bogaty P, Pibarot P. Paradoxical low flow, low gradient severe aortic stenosis despite preserved ejection fraction is associated with higher afterload and reduced survival. Circulation. 2007;115:2856-64.

5. Antonini-Canterin F, Huang G, Cervesato E, Faggiano P, Pavan D, Piazza R, et al. Symptomatic aortic stenosis: does systemic hypertension play an additional role? Hypertension. 2003:41:1268-72.

6. Vahanian A, Alfieri O, Andreotti A, Antunes MJ, Barón-Esquivias G, Baumgartner $\mathrm{H}$ et al. Guidelines on the management of valvular heart disease (version 2012): The Joint Task Force on the Management of valvular heart disease of the European society of cardiology (ESC) the European Association for Cardio-Thoracic Surgery (EACTS). Eur Heart J. 2012;33:2451-96.

7. Bonow RO, Carabello BA, Chatterjee K, De Leon Jr AC, Faxon DP, Freed MD, et al; American College of Cardiology/American Heart Association Task Force on Practice Guidelines. 2008 focused update incorporated into the ACC/AHA 2006 guidelines for the management of patients with valvular heart disease: a report of the American College of Cardiology/American Heart Association Task Force on Practice Guidelines (Writing Committee to revise the 1998 guidelines for the management of patients with valvular heart disease). Endorsed by the Society of Cardiovascular Anesthesiologists, Society for Cardiovascular Angiography and Interventions, and Society of Thoracic Surgeons. J Am Coll Cardiol. 2008;52:e1-142.

8. Minners J, Allgeier M, Gohlke-Baerwolf C, Kienzle RP, Neumann FJ, Jander N. Inconsistencies of echocardiographic criteria for the grading of aortic valve stenosis. Eur Heart J. 2008;29:1043-8.

9. Lancellotti P, Donal E, Magne J, O’Connor K, Moonen ML, Cosyns B, et al. Impact of global left ventricular afterload on left ventricular function in asymptomatic severe aortic stenosis: a two-dimensional speckle-tracking study. Eur J Echocardiogr. 2010;11:537-43.

10. Herrmann S, Stork S, Niemann M, Lange V, Strotmann JM, Frantz S, et al. Lowgradient aortic valve stenosis myocardial fibrosis and its influence on function and outcome. J Am Coll Cardiol. 2011;58:402-12.

11. Barasch E, Fan D, Chukwu EO, Han J, Passick M, Petillo F, et al. Severe isolated aortic stenosis with normal left ventricular systolic function and low transvalvular gradients: pathophysiologic and prognostic insights. J Heart Valve Dis. 2008;17:81-8.

12. Clavel MA, Dumesnil JG, Capoulade R, Mathiew P, Senechal M, Pibarot P. Outcome of patients with aortic stenosis, small valve area, and low-flow, low-gradient despite preserved left ventricular ejection fraction. J Am Coll Cardiol. 2012;60:1259-67.

13. Rosenhek R, Binder T, Porenta G, Lang I, Christ G, Schemper M, et al. Predictors of outcome in severe, asymptomatic aortic stenosis. N Engl J Med. 2000;343: 611-7.

14. Lancellotti P, Magne J, Donal E, Davin L, O’Connor K, Rosca M, et al. Clinical outcome in asymptomatic severe aortic stenosis: insights from the new proposed aortic stenosis grading classification. J Am Coll Cardiol. 2012;59: 235-43.

15. Monin JL, Lancellotti P, Monchi M, Lim P, Weiss E, Pierard LA, et al. Risk score for predicting outcome in patients with asymptomatic aortic stenosis. Circulation. 2009;120:69-75.

16. Melis G, Frontera G, Caldentey G, Sahuquillo A, Fernández-Palomeque C, Forteza JF, et al. El análisis del flujo aórtico por ecocardiografía Doppler es útil en la estratificación pronóstica de los pacientes con estenosis aórtica grave con fracción de eyección normal. Rev Esp Cardiol. 2013;66:261-8. 